\section{Proton-Transfer Reactions}

Edited by E. F. CALDIN and V. GOLD, F.R.S. 1975: 456 pages: illustrated: 412127008 : hardback: $f 18.00$

Many of the fundamental concepts of chemistry - from the tunnel effect in chemical kinetics to catalysis by enzymes - owe their recognition or formulation to the study of protontransfer reactions. The subject has been developed in extensive researches by many workers, and most notably by Professor R. P. Bell, whose book The Proton in Chemistry has become a modern classic of science. The present volume, dedicated to Professor Bell, aims to complement his book by providing a coordinated set of studies in depth on some of the central problems and growing-points of proton-transfer reactions. written by a group of leading workers in the field

\section{Second Edition

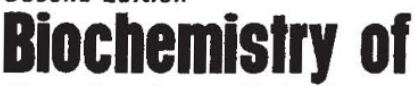 Antimicrobial Action}

T. J. FRANKLIN and G. A. SNOW

Hardback: Second edition 1975: 240 pages: 7 tone and 78 line illustrations: 412129000 : f7.00

Science Paperback: 41212910 8: $f 3.95$

This book provides a systematic guide to the field of antimicrobial biochemistry. The mechanisms by which antimicrobial agents have their effect are illustrated by a large number of examples, leading to a discussion of the development of drug resistance in microorganisms. Extensive revisions have been made in this second edition and these include substantial changes in virtually every chapter. A new chapter has been added on the penetration of antimicrobial agents into cells.

\section{Second Edition}

\section{Lipid Biochemistry}

M. I. GURR and A. T. JAMES

Hardback: Second edition November 1975: 256 pages: illustrated: 41213780 1: f6.50 Science Paperback: 41213790 9: $£ 3.95$

This new edition has been thoroughly updated and the authors have added new material where appropriate - as, for example, the latest knowledge on the pathway for the biosynthesis of ether bonds. A new chapter Lipids in food, has also been added for the new edition. This chapter not only covers the biochemistry and physiology of dietary fats but also their role in the texture, flavour and aroma of foods. It also includes a discussion of diseases (heart disease, obesity, diabetes) in which dietary fats are thought to play a role.

CHAPMAN \& HALI

11 New Fetter Lane, London EC4P 4EE

Circle No. 11 on Reader Enquiry Form.

\section{Dynamic molecules}

Molecular Reaction Dynamics. By R. D. Levine and R. B. Bernstein. Pp. vi+150. (Clarendon: Oxford; Oxford University Press: New York, November 1975.) $£ 5.00$.

THE last fifteen years have seen rapid progress in one of the most fundamental areas of chemical researchthe awakening of our understanding of how reactions occur at the microscopic level. Indeed much of this work is so recent that an adequate grasp of the field can only be acquired after extensive perusal of the original literature and the relatively limited number of research reviews. It is for this reason that the appearance of Levine and Bernstein's Molecular Research Dynamics is particularly welcome at this time.

The book is directed at 'newcomers' to the field and contains material which has been presented orally to advanced undergraduates and, presumably, graduate students. The contents are organised into six chapters which make up a total of some 30 sections, most of which are subdivided further. The avowed aim is to provide a general feeling for the whole subject area rather than supply extensive detailed knowledge, and as such the volume is, as it sets out to be, much more of a "course book' than a reference work. Chapter 1 serves to whet the appetite for the study of molecular dynamics, after which the logic is to initiate a detailed consideration of molecular collisions (chapter 2) as a prelude to an account of how scattering phenomena can be related to collision dynamics (chapter 3 ). This leads to a consideration of the trajectories across potential energy surfaces, the theoretical calculation of reaction rates, the relationship between microand macroscopic observations, the partitioning between the different forms of energy in the products (chapter 4) and a survey of the different forms of molecular energy transfer (chapter 5). The climax of this development is then experienced in chapter 6 in which the previous extensive preparation is applied to the experimental study and discussion of a series of chemical systems exhibiting respectively different types of interaction between the reacting species. The various sections of the book are adequately cross-referenced forwards and backwards.

The method of presentation is such that maximum value can only be derived from the text by reading it cover-to-cover a number of times, and a newcomer would only acquire a real understanding of some of the mathematical material after a prolonged study of the relevant published literature. An annoying and distracting feature, which seems to be characteristic generally of 'beam' publications, is the extensive use of footnotes, often containing asides or amplifications of the discussion, which could readily be either omitted or incorporated into the main text. Apart these objections, however, the book undoubtedly satisfies the need for a readable and comprehensive guide to this important area of study at an attractive price. J. F. J. Todd

\section{Numbers}

Transcendental Number Theory. By Alan Baker. Pp. $\mathrm{x}+147$. (Cambridge University Press: London, April 1975.) £4.90; $\$ 13.95$.

A NUMBER is said to be algebraic if it is a root of a non-zero polynomial in one variable, the coefficients of which are integers, and to be transcendental otherwise. It is well-known that (in a measure theoretic sense) almost all numbers are transcendental, but to demonstrate that a specific number is transcendental is never trivial. The methods for solving this problem constitute transcendental number theory.

Professor Baker's tract is an account of the advances in this subject during the last decade. A short description of the origins of the theory is followed by an amalgam of the author's award-winning papers (Fields Medal) on linear forms in logarithms with some very interesting applications both in transcendence theory and in such other areas as Diophantine equations and Gauss's conjecture on discriminants of class number one. Further topics include Schmidt's theorem on simultaneous approximation of several algebraic numbers by rationals, Mahler's classification of transcendental numbers and Shidlovsky's theorem on algebraic independence.

The proofs of the major theorems are presented in a very economic style and can be fully understood only by students with a plentiful supply of pencil, paper and time. For such serious students the book will form an admirable introduction to this difficult field of research, being in addition a concise source book for the major additional developments and outstanding problems. Those wishing to obtain an appreciation of the scope of this field will also gain much from the more descriptive parts of the work. All readers of this timely and welcome tract will benefit from the appraisal of a pre-eminent contributor to the field.

D. A. Burgess 